Chaverra-Fernández, B.; Hernández-Álvarez, J.L. (2021) Metaevaluation in Physical Education Teachers. Revista Internacional de Medicina y Ciencias de la Actividad Física y el Deporte vol. 21 (84) pp. 501-513. Http://cdeporte.rediris.es/revista/revista83/artmetaevaluacion1270.htm DOI: https://doi.org/10.15366/rimcafd2021.83.006

\title{
ORIGINAL
}

\section{LA METAEVALUACIÓN EN EL PROFESORADO DE EDUCACIÓN FÍSICA}

\section{METAEVALUATION IN PHYSICAL EDUCATION TEACHERS}

\author{
Chaverra-Fernández, B. ${ }^{1}$ y Hernández-Álvarez, J.L. ${ }^{2}$ \\ ${ }^{1}$ Doctora en Ciencias de la Actividad Física y del Deporte. Docente de tiempo completo de la \\ Universidad de Antioquia (Colombia) beatriz.chaverra@udea.edu.co \\ ${ }^{2}$ Doctor en Filosofía y Ciencias de la Educación. Catedrático e investigador de la Universidad \\ Autónoma de Madrid (España) juanluis.hernandez@uam.es
}

Código UNESCO I UNESCO code: 5899 Otras Especialidades Pedagógicas (Educación Física y Deporte) / Other pedagogical specialties (Physical Education and Sport)

Clasificación Consejo de Europa / Council of Europe classification: 5. Didáctica y metodología / Didactic and methodology

Recibido 9 de julio de 2019 Received July 9, 2019

Aceptado 7 de marzo de 2020 Accepted March 7, 2020

\section{RESUMEN}

Este artículo presenta resultados de una investigación más amplia sobre las concepciones y la acción evaluativa de seis docentes de educación física de Medellín (Colombia). El objetivo específico que se presenta fue: Analizar las reflexiones que pueden surgir en el profesorado una vez finalizado el proceso evaluativo en una unidad didáctica, es decir, la evaluación de la propia evaluación (metaevaluación). El diseño utilizado fue cualitativo, específicamente un estudio de caso múltiple. Se utilizaron como estrategias de recolección de información las entrevistas semi-estructuradas y, cuando fue posible por su existencia, el análisis documental. La investigación permite concluir que, finalizar una unidad didáctica no conlleva necesariamente a una reflexión del profesorado sobre la evaluación realizada. Los pocos cuestionamientos mencionados en sus discursos, se refieren a breves reflexiones sobre los instrumentos 0 procedimientos realizados, pero no hay una reflexión profunda sobre los propósitos formativos que tiene la evaluación y sobre sus aportes a una enseñanza de calidad.

PALABRAS CLAVE: educación física, metaevaluación, pensamiento del 
profesor, evaluación formativa.

\begin{abstract}
This article presents the results of a broader research on the conceptions and evaluative action of six physical education teachers in high school in Medellin (Colombia). This paper presents the findings on one of its objectives: Analyze the reflections that can arise in the teaching staff once the assessment process is completed in a didactic unit, that is, the assessment of the assessment-self (meta-evaluation). The design used was qualitative, specifically a multiple case study. The strategies used were semi-structured interviews and documentary analysis. The research allows us to conclude that the moment after the didactic, does not suggest a reflection in the teachers about the assessment. The few questions mentioned in his discourse refer to the instruments or procedures performed, but there is no analysis of the purposes of the assessment and its contributions to quality.
\end{abstract}

KEY WORDS: physical education, meta-evaluation, teacher thinking, formative assessment.

\title{
INTRODUCCIÓN
}

La evaluación es un tema álgido en la educación, porque en ella confluyen múltiples miradas, funciones y concepciones. Algunos autores la han considerado como el aspecto más difícil de abordar en la educación (Cabezas, González, \& Carpintero, 2009; Cerda, 2000; Salinas, 2002; Santos, 2003) y no se han equivocado.

La complejidad, desde nuestra perspectiva, se enmarca en la subjetividad que se refleja en cada acción evaluativa. Es decir, la concepción que tenga el profesor sobre la evaluación se verá reflejada en su discurso, en el nivel de participación de los estudiantes, en la relación con la calificación, en el sentido de los instrumentos evaluativos, en los momentos en que evalúa, etc. Por tanto, como lo plantea Brown (2002), es necesario profundizar en la relación entre el pensamiento y la acción en el profesorado y desde allí identificar el porqué de sus prácticas y proponer acciones de mejora.

Este artículo presenta las reflexiones post-interactivas del profesorado participante sobre la evaluación al finalizar una unidad didáctica. En términos generales, la fase post-interactiva de la enseñanza, denominada así inicialmente por Jackson (1968), se refiere al momento en que el profesorado tiene la oportunidad de evaluar cómo fue su proceso educativo y plantear alternativas que mejoren su enseñanza y el aprendizaje del estudiantado, lo que a la vez, se constituye en un insumo para iniciar nuevamente otro ciclo educativo. En definitiva, "Se trata de una fase en la que el docente debe interrogarse sobre las consecuencias de las decisiones y acciones que ha puesto en práctica" (Hernández \& López, 2004, p. 61). 
La enseñanza reflexiva o práctica reflexiva ha sido abordada por diversos autores, que rescatan sus cualidades y aportes a la calidad educativa. Para Jinhong (2012) la enseñanza reflexiva es el acto de pensar, analizar o evaluar los significados, intenciones, creencias, decisiones, acciones o productos educativos que se obtienen a través de esos procesos de pensamiento.

Zeichner y Tabachnick (1991, citados por Zeichner, 1998) presentan cuatro tradiciones de la práctica reflexiva que han sido utilizadas en la enseñanza y en la formación del profesorado en Estados Unidos: la académica, la eficiencia social, la desarrollista y la reconstruccionista social. Cada una conlleva una apuesta política y un énfasis particular. Sin embargo, como lo resalta Zeichner, no es posible encontrar una tradición pura, por el contrario, los programas de formación del profesorado y los propios maestros, pueden llevar a cabo una mezcla de elementos en las que se da mayor valor a unas características de la tradición que a otras.

De igual forma, la afirmación de Van Manen (1977) diferenciando tres niveles de reflexión sigue teniendo plena vigencia en la actualidad. El primero es más técnico, donde la reflexión se centra en la selección y el uso adecuado de las estrategias didácticas que el maestro va a utilizar en el aula y donde prima una preocupación por los medios y no por los fines de la enseñanza. En el segundo nivel, la atención se centra en la comprensión interpretativa de la calidad de la experiencia educativa y de tomar decisiones prácticas. Es decir, la reflexión se realiza sobre las consecuencias de determinadas estrategias. Por último, el tercer nivel, asume la reflexión desde la postura autocrítica e incorpora aspectos morales, éticos y políticos de la enseñanza y la educación. En este nivel, la reflexión integra un análisis crítico de las instituciones y de la autoridad.

Por otro lado, Schön (1998) se refiere a la reflexión desde otra perspectiva, la denomina reflexión sobre la acción, es decir; el análisis que tiene lugar una vez finalizada la propia acción educativa. Para él, esa reflexión se constituye en un paso determinante para el aprendizaje permanente del profesorado y el insumo privilegiado para transformar la propia práctica.

En el campo educativo existen diversas investigaciones sobre la importancia de la reflexión en el profesorado, pero sólo una pequeña parte de estas investigaciones se han realizado en educación física (Dervent, 2015; Jinhong, 2012; Tristán, 2010; Tsangaridou, 2005). Los estudios en el área han aparecido esporádicamente convirtiéndose en un interés creciente de investigación (Hall \& Smith, 2006).

Si llevamos esta teoría de la reflexión sobre la acción al campo evaluativo, podemos hablar de una metaevaluación, es decir, la evaluación que realiza el profesorado sobre su acción evaluativa. El término metaevaluación fue utilizado por primera vez por Scrive en 1969 (citado por Stufflebeam, 2001) quien definía la metaevaluación "como toda evaluación de una evaluación, sistema de evaluación, o un dispositivo de evaluación" (p. 185).

Esta evaluación debe asumirse como una acción comprensiva que le permite al 
maestro pensar sobre sí mismo y reflexionar sobre la acción realizada.

...el propósito de la metaevaluación es la explicación de la evaluación como un proceso social complejo. Se refiere al abordaje del objeto -la evaluación- lidiando directamente con la complejidad no sólo como red de acciones sociales sino también en sus referentes y explicaciones de carácter axiológico, ideológico y epistemológico, fundamentalmente (Díaz, 2001, p. 175).

Si tenemos presente que la evaluación permite obtener información sobre el proceso de enseñanza-aprendizaje realizado, para tomar decisiones al respecto, se asume que la evaluación no se realiza como un acto mecánico, sino que entraña una razón pedagógica que la sustenta, con el interés fundamental que los estudiantes aprendan más y que el profesorado perfeccione su práctica docente (Barrientos, López-Pastor, \& Pérez-Brunicardi, 2019; López-Pastor, Molina-Soria, Pascual-Arias, \& Manrique-Arribas, 2020; Trigueros-Cervantes, Rivera-García, \& De la Torre-Navarro, 2012; Velázquez \& Hernández, 2004). Desde esta perspectiva, una de las preguntas que puede plantearse la metaevaluación es precisamente sobre la potencialidad que ha tenido la evaluación para promover cambios (Santos \& Moreno, 2004).

Los estudios realizados por Jiménez, Navarro y Jiménez (2001), Jiménez y Navarro (2008), Navarro y Jiménez (2012) y Cano y Ruiz (2019) constituyen algunos de los pocos referentes de metaevaluación en educación física escolar. La investigación llevada a cabo por Navarro y Jiménez (2012) presenta la metaevaluación como un fuerte proceso que permite la reflexión y transformación de la acción evaluativa. Los investigadores emplearon un instrumento de metaevaluación didáctica con cuatro maestros con el fin de diagnosticar el alcance formativo de la evaluación que emplean en sus unidades didácticas. "Este instrumento de autodiagnóstico aporta a los maestros las claves operativas con las que reconocer los límites formativos del modelo de evaluación empleado y activar el cambio en su intervención docente" (Navarro \& Jiménez, 2012, p. 75).

Los resultados del estudio muestran cómo a partir del autodiagnóstico y mediado por un proceso de reflexión conjunta, los maestros han encontrado las claves para mejorar su proceso evaluativo desde una perspectiva formativa, especialmente centrado en la implicación del alumnado en la evaluación de su aprendizaje, lo cual fue confirmado por los mismos estudiantes a través de un cuestionario. Estos estudios dejan abierta la necesidad de profundizar en la metaevaluación como la posibilidad de reflexionar y mejorar las prácticas evaluativas que lleva a cabo el profesorado.

En consecuencia, el objetivo de esta investigación fue analizar qué piensa el profesorado sobre la evaluación realizada una vez finalizada la unidad didáctica, qué cuestionamientos surgen sobre su propio desempeño, cómo proyecta su evaluación en unidades futuras y cómo evalúa su propia evaluación. 


\section{DESARROLLO DE LA INVESTIGACIÓN}

La investigación se llevó a cabo con un diseño cualitativo. En palabras de Taylor y Bogdan (1987), la metodología cualitativa es más que un conjunto de técnicas para recoger datos "es un modo de encarar el mundo empírico" (p. 20), es decir; la investigación desde la perspectiva cualitativa, constituye una forma de comprender el mundo, una manera particular de acercarse a las realidades (Galeano, 2004).

Se eligió como diseño el estudio de caso múltiple (Chaverra-Fernández, GaviriaCortés, \& González-Palacio, 2019; Stake, 2006) de tipo instrumental y descriptivo. Los participantes fueron seis docentes de educación física, cuatro hombres (Juan, Carlos, Luis y Pedro) y dos mujeres (Diana y María) y con diversidad de años de experiencia docente (Entre 10 y 20 años). La selección de los participantes fue basada en criterios (Goetz \& LeCompte, 1988), los cuales fueron: tener una titulación profesional en Educación Física, tener mínimo cinco años de experiencia como docentes de educación física, ser profesores activos en una institución educativa pública en la ciudad y tener disposición y tiempo para participar del estudio.

Las estrategias de recolección de información fue, principalmente, las entrevistas semi-estructuradas (Massot, Dorio, \& Sabariego, 2014) y, cuando fue posible por su existencia, el análisis documental (Bowen, 2009; Fitzgerald, 2007). Las entrevistas permitieron descubrir desde los propios relatos del profesorado, las reflexiones que pueden surgir sobre la evaluación realizada o metaevaluación. Para su realización se construyó un guion validado por un grupo de tres profesores doctores en educación física con experiencia investigativa en evaluación y una doctora en educación no especialista en educación física. El objetivo de este ejercicio fue evaluar la claridad y pertinencia de las preguntas formuladas y así validar el instrumento.

El análisis documental, como técnica complementaria, se realizó sobre textos oficiales (Del Rincón, Arnal, Latorre, \& Sans, 1995; Woods, 1987), es decir, los documentos que determina cada colegio. Específicamente, se analizaron las carpetas institucionales de tres docentes participantes. Esta carpeta contiene varios formatos oficiales, entre ellos, uno denominado reflexiones pedagógicas. Allí cada docente puede realizar los comentarios y reflexiones que considere pertinentes sobre el desarrollo de la unidad didáctica. Los colegios de los otros profesores participantes no tenían documentos oficiales que pudieran ser fuente de información y análisis, por lo que en las entrevistas se trató de indagar sobre aspectos que podrían, de haber existido, estar incluidos en dichos documentos.

Estos documentos permitieron contrastar la información recolectada en las entrevistas y aumentar la credibilidad de la investigación. A la vez, el acceso a estos documentos proporcionó una mejor lectura del contexto institucional en que se desenvuelve cada docente.

La información fue analizada a partir de las tres fases propuestas por Taylor y 
Bogdan (1987). En la fase de descubrimiento se identificaron categorías y temas a partir de la lectura minuciosa de las entrevistas. En esta lectura, se fueron registrando conceptos, ideas y reflexiones en función al objetivo de la investigación.

En la fase de codificación se expandieron o descartaron las categorías iniciales, dado que a partir del análisis de la información y la lectura del contexto, surgían categorías de primer y segundo nivel de acuerdo a los objetivos del estudio. La información se analizó de manera inductiva (Bonilla \& Rodríguez, 1997), es decir, las categorías emergieron a partir de las recurrencias en los datos. Se crearon los códigos para la lectura de los resultados de acuerdo a la estrategia y la letra inicial del seudónimo del docente, ejemplo: E.3.M (Entrevista tres de María), Cl.J (Carpeta institucional de Juan).

En la fase de relativización de los datos (nombrada así por Deutsher \& Mills, 1940, citados por Taylor \& Bogdan 1987); se buscó no generalizar las afirmaciones realizadas por los docentes, sino comprenderlas desde la realidad de su contexto y analizarlas a partir de los fundamentos teóricos e investigaciones al respecto.

Desde el punto de vista metodológico, la legitimación del conocimiento construido en la investigación cualitativa se fundamenta en el diálogo y la intersubjetividad. En este sentido, la fiabilidad, objetividad y validez tienen un significado diferente en la investigación cualitativa al otorgado en el enfoque positivista que sustenta un buen número de las investigaciones cuantitativas (Guba, 1989; Rodríguez, Gil, \& García, 1999). Para esta investigación se asumieron los términos naturalistas que propone Guba (1989): credibilidad, transferibilidad, dependencia y confirmabilidad. En este caso se realizaron diversas acciones como la triangulación de la información (entrevistas, análisis documental y teoría e investigaciones sobre el tema), la comprobación con los participantes de los análisis realizados y la revisión de varios investigadores.

Las consideraciones éticas del estudio fueron: El consentimiento informado, la confidencialidad de la información, el uso de seudónimos y la devolución de la información a los participantes.

\section{RESULTADOS}

Como se ha mencionado anteriormente, esta investigación presenta resultados parciales de un estudio más amplio sobre las concepciones y la acción evaluativa del profesorado (Chaverra-Fernández, 2017). Durante la investigación se realizaron diversas entrevistas que buscaban conocer las concepciones y acciones evaluativas de los participantes. En esas conversaciones, los relatos del profesorado fueron extensos y cargados de ejemplos y anécdotas. Por el contrario, la entrevista que indagó por los cuestionamientos o reflexiones sobre lo ocurrido durante la unidad, fueron cortas, con pocas opciones de profundizar en sus respuestas.

Las escasas palabras del profesorado, pueden asumirse como un reflejo de lo 
difícil que es reflexionar sobre la propia actuación como maestros, o la poca costumbre que se tiene de cuestionar la propia experiencia (ChaverraFernández, 2017).

La percepción general del profesorado sobre su desempeño durante la unidad didáctica fue positiva. Aunque durante la investigación fue difícil conocer con precisión cuáles eran sus objetivos de aprendizaje y sus criterios de evaluación, expresan tranquilidad por haber realizado un buen trabajo y satisfacción, precisamente, por los logros obtenidos en el estudiantado.

... los objetivos se cumplieron... me parece que se lograron muchas cosas, uno queda tranquilo cuando se logran los objetivos. (E.3.C.)

Los profesores Juan y Pedro dejaron plasmada su percepción positiva de la enseñanza en el formato de reflexiones pedagógicas, allí cada docente puede realizar los comentarios que considere pertinentes sobre el desarrollo de la unidad didáctica.

Me voy con la sensación de haber hecho un buen trabajo, pero también aprendí muchas cosas de mis estudiantes. (Cl.J.)

... a pesar de los días que no se pudo dar las clases por diferentes actividades planeadas en la institución, se pudo lograr las metas. (CI.P.)

La apreciación positiva del desempeño, está muy ligada a una percepción de aprendizaje del estudiantado, es decir, consideran que hubo un aprendizaje en ellos y, por tanto, la autoevaluación que realizan a su labor docente es positiva.

... siento que la clase estuvo bien, que ellos aprendieron, que se sintieron bien, entonces yo me siento bien con el aprendizaje que ellos tuvieron. (E.3.L.)

En términos generales, el profesorado considera que realizó las acciones evaluativas que tenía previstas. Sin embargo, se percibe en su discurso que sus reflexiones se mueven entre lo realizado en la unidad que terminó y las acciones que tienen establecidas desde hace algún tiempo en la enseñanza. Esa coherencia les brinda seguridad para asegurar que su acción evaluativa estuvo acorde a sus expectativas.

... lo que yo había pensado y como evalúo es así, teniendo en cuenta participación, interés en la clase, responsabilidad. (E.3.L.)

... eso siempre lo hago... son todos los componentes que debe tener (la evaluación): la parte teórica, la parte física, la parte actitudinal y la autoevaluación de ellos. (E.3.C.)

En consecuencia, la valoración positiva de la acción evaluativa se sustenta sobre la percepción de haber cumplido con las rutinas establecidas, pero la conciencia crítica, para plantearse si esas rutinas son realmente las más adecuadas para 
incidir de manera positiva sobre el proceso de aprendizaje del estudiantado, es limitada. Igualmente, la reflexión sobre la calidad y adecuación de los propios objetivos de aprendizaje es muy escasa.

A pesar de cumplir con sus expectativas y manifestar una satisfacción con el proceso evaluativo realizado, algunos profesores reconocen que hay aspectos que pueden mejorar. Específicamente Carlos y Diana señalan dos acciones que les gustaría mejorar en sus próximas unidades didácticas.

... otra cosa que yo le agregaría, que siempre he querido hacerlo, es la evaluación teórica. (E.3.C.)

... yo haría una sensibilización más profunda frente a la evaluación... pero no una evaluación de la nota (calificación), sino del proceso. (E.3.D.)

Los cambios propuestos, o la posibilidad de hacerlos, se enmarcan en aspectos formales de la acción de evaluar, pero no se orientan a cambios en la finalidad, contenidos o actores de la evaluación. No se encuentra en los participantes un cuestionamiento profundo sobre el valor formativo de la evaluación realizada 0 cómo mejorarla.

Otro tema que surge de manera tímida en sus discursos es sobre los instrumentos utilizados, no con el propósito de modificarlos o aplicar otros nuevos, sino para ratificarlos. Pedro y María destacan la necesidad de continuar con el uso de la planilla de seguimiento como instrumento principal de registro de calificaciones, ya que es una prueba vital de la evaluación realizada para el estudiante, el padre de familia y el colegio. Es importante señalar que la planilla es un formato donde el profesorado registra la asistencia y calificaciones de cada clase, pero no contiene criterios concretos de evaluación.

... con las planillas hay que seguir, eso no se puede cambiar. (E.3.M.)

... hay que manejar planilla porque llega un padre de familia y yo necesito darle una nota. (E.3.P.)

El uso de la planilla de seguimiento responde así a la institucionalización del proceso de evaluación y a la necesidad de justificación ante el colegio y las familias. El propio instrumento está condicionado por esa institucionalización de la calificación, pero no existe un cuestionamiento docente sobre el contenido de la plantilla y su posible valor para el proceso de evaluación formativa. De hecho, en algún caso, se pone de manifiesto una presión sobre las calificaciones que no contribuye a una evaluación serena, tal y como reconoce María, que a través de una reflexión crítica denuncia la obsesión por la calificación obligada por la normativa de uso de la plantilla:

Regular también (Evaluación en la unidad)...hubo momentos en los que yo sacaba notas porque era obligación sacarlas... que así llueva o truene usted tiene que sacar una nota, así usted dé el contenido como es, así no lo dé, por pararse, por reírse, por hablar, por jugar, por no jugar, pero 
sáquela, o sea acostúmbrese María a que por lo que sea sáquela... (E.3.M.)

El único profesor que encuentra en un instrumento un aporte valioso para mejorar su labor como maestro es Juan. Para él, la información que le brinda el cuaderno del estudiante, es un insumo para modificar su acción en unidades futuras.

... yo creo mucho en esos cuadernos, porque finalmente es el registro de lo que ellos hacen... yo los reviso y me proyecto: bueno para la próxima unidad con ese grupo o con el grado puedo hacer esto, o modifico esto, digamos que esto fue provechoso. (E.3.J.)

El análisis global de lo declarado por el profesorado, nos induce a pensar que, la valoración positiva de la acción evaluativa se sustenta sobre la percepción de haber cumplido con las rutinas de evaluación que tienen establecidas hace algunos años, pero la actitud crítica, para plantearse si esas rutinas son realmente las más adecuadas y, si inciden de manera positiva sobre el proceso de aprendizaje del estudiantado, se refleja de forma muy tímida en sus comentarios.

\section{DISCUSION Y CONCLUSIONES}

Si bien la reflexión es una tarea compleja, las ideas presentadas por el profesorado participante se quedan en el primer nivel que propone Van Manen (1977) o en la tradición académica de Zeichner (1998). Un nivel técnico donde los pensamientos se centran en los medios y no en los fines de la enseñanza. En este caso, no se encuentra una reflexión sobre los propósitos de la evaluación y sus aportes para mejorar la docencia, sino que sus pocos pensamientos se centran en los procedimientos realizados y el deseo de cambiar algunas estrategias e instrumentos.

Las ideas expresadas por el profesorado, reflejan la escasa reflexión sobre la evaluación realizada. Sin duda, la capacidad de pensar sobre la propia acción no es un hecho esporádico al que se llega sólo con el paso de los años, sino que debe existir una formación y actitud crítica que predisponga a pensar por qué hacen lo que hacen y contar con habilidades para hacerlo.

Estas habilidades son una deuda de la formación inicial y permanente, ya que como lo plantea Zeichner (2008), la formación docente ha hecho muy poco para fomentar la reflexión en el profesorado; ha olvidado dotarlo de habilidades para aprender de sus experiencias y valerse de ese conocimiento para ser mejores en aquello que hacen a lo largo de sus carreras docentes.

Tsangaridou (2005) afirma que, el conocimiento del contexto, del contenido y del estudiantado, son requisitos previos para facilitar un proceso de reflexión en el profesorado. Sin embargo, los participantes de esta investigación tienen un amplio conocimiento de los tres elementos y, aun así, no se identifica una reflexión profunda sobre su desempeño, lo que indica que hace falta un elemento 
adicional a los tres que menciona el autor: una formación hacia la reflexión que permita pasar de la microreflexión a la macroreflexión (Tsangaridou \& O'Sullivan, 1997).

Por otro lado, diversas investigaciones han demostrado que la aplicación de un instrumento de autodiagnóstico y la participación en un proceso de reflexión teórica sobre la evaluación, contribuye a que los docentes encuentren algunas claves para mejorar su proceso evaluativo desde un enfoque formativo (Cano \& Ruiz, 2019; Jiménez \& Navarro, 2008; Jiménez et al., 2001; Navarro \& Jiménez, 2012). En esta perspectiva, es posible pensar que se puede potenciar la reflexión en el profesorado a partir del acompañamiento, la formación y la búsqueda de sus propios caminos y necesidades de acuerdo a los contextos en que se desenvuelven.

En conclusión, podemos afirmar que, la metaevaluación no es una acción que motive al profesorado, bien sea por desconocimiento o por no contar con las habilidades necesarias para realizarla. La muy escasa reflexión del profesorado sobre la evaluación pone la atención en los instrumentos utilizados, pero no existen referencias a los propósitos que tienen con la evaluación o cómo su análisis (meta-evaluación) podría servirles para mejorar su enseñanza. Sin duda, es necesario que los programas de formación docente incidan no solo en el desarrollo de conocimientos y habilidades para llevar a cabo un proceso de metaevaluación, sino también en la disposición o actitud de los docentes hacia esta práctica imprescindible para la mejora de la educación y del propio desarrollo profesional.

\section{REFERENCIAS BIBLIOGRÁFICAS}

Barrientos, E., López-Pastor, V., \& Pérez-Brunicardi, D. (2019). ¿Por qué hago evaluación formativa y compartida y/o evaluación para el aprendizaje en EF? La influencia de la formación inicial y permanente del profesorado. Retos. Nuevas tendencias en Educación Fisica, Deporte y Recreación, 36(20 semestre), 37-43. https://doi.org/10.47197/retos.v36i36.66478

Bonilla, E., \& Rodríguez, P. (1997). Más allá del dilema de los métodos: la investigación en ciencias sociales. Bogotá: Norma.

Bowen, G. (2009). Document analysis as a qualitative research method. Qualitative Research Journal, 9(2), 27-40. https://doi.org/10.3316/QRJ0902027

Brown, G. (2002). Teachers' Conceptions of Assessment. (Tesis Doctoral), University of Auckland, Auckland.

Cabezas, D., González, C., \& Carpintero, E. (2009). Evaluar la evaluación: cuestionario sobre prácticas de evaluación. EduPsykhé: Revista de psicología y educación, 8(1), 51-61.

Cano, A., \& Ruiz, E. (2019). Diagnóstico y evolución hacia un modelo de evaluación formativa en maestros de educación física en primaria. Infancia, Educación $\quad y \quad$ Aprendizaje, $5(2), \quad$ 496-503. https://doi.org/https://doi.org/10.22370/ieya.2019.5.2.1710

Cerda, H. (2000). La evaluación como experiencia total. Logros-objetivosprocesos- competencias y desempeño. Bogotá: Cooperativa Editorial 
Magisterio.

Chaverra-Fernández, B. (2017). Pensamiento y acción evaluativa del profesorado de educación física de educación secundaria y media: Estudio de casos en Medellín-Colombia. (Tesis doctoral). Universidad Autónoma de Madrid, Madrid.

Chaverra-Fernández, B., Gaviria-Cortés, D., \& González-Palacio, E. (2019). El estudio de caso como alternativa metodológica en la investigación en educación física, deporte y actividad física. Conceptualización y aplicación. Retos. Nuevas tendencias en Educación Fisica, Deporte y Recreación, $1^{\circ}$ semestr(35), 371-377. https://doi.org/10.47197/retos.v0i35.60168

Del Rincón, D., Arnal, J., Latorre, A., \& Sans, A. (1995). Técnicas de investigación en ciencias sociales. Madrid: Dykinson.

Dervent, F. (2015). The effect of reflective thinking on the teaching practices of preservice physical education teachers. Issues in Educational Research, 25(2), 260-275.

Díaz, L. (2001). La metaevaluación y su método. Revista de Ciencias Sociales (Cr), II-III(93), 171-192.

Fitzgerald, T. (2007). Documents and documentary analysis: reading between the lines. En A. Briggs \& M. Coleman (Eds.), Research methods in educational leadership and management (2०, pp. 278-294).

Galeano, M. (2004). Estrategias de investigación social cualitativa. El giro de la mirada. Medellín: La Carreta.

Goetz, J., \& LeCompte, M. (1988). Etnografía y diseño cualitativo en investigación educativa. Madrid: Morata.

Guba, E. (1989). Criterios de credibilidad en la investigación naturalista. En J. Gimeno \& A. Pérez (Eds.), La enseñanza, su teoría y su práctica (pp. 148165). Madrid: Akal.

Hall, T., \& Smith, M. (2006). Teacher Planning, Instruction and Reflection: What We Know About Teacher Cognitive Processes. Quest, 58(4), 424-442. https://doi.org/10.1080/00336297.2006.10491892

Hernández, J., \& López, A. (2004). Evaluación de la enseñanza: análisis y propuestas. En J. Hernández \& R. Velásquez (Eds.), La evaluación en educación física. Investigación y práctica en el ámbito escolar (pp. 49-76). Barcelona: Graó.

Jackson, P. (1968). Life in classrooms. New York: Holt, Rinhart y Winston.

Jiménez, F., \& Navarro, V. (2008). Evaluación formativa y metaevaluación en educación física: dos estudios de casos colectivos en las etapas de educación primaria y secundaria. Revista Española de Educación Física y Deportes, 9(Julio-Diciembre), 13-25.

Jiménez, F., Navarro, V., \& Jiménez, H. (2001). Incidencia de la metaevaluación en la evaluación formativa de la educación física. Evaluación e intervención psicoeducativa: Revista Interuniversitaria de Psicología de la Educación, 6 y $7(I), 259-274$.

Jinhong, J. (2012). The focus, role, and meaning of experienced teachers' reflection in physical education. Physical Education and Sport Pedagogy, 17(2), 157-175. https://doi.org/10.1080/17408989.2011.565471

López-Pastor, V., Molina-Soria, M., Pascual-Arias, C., \& Manrique-Arribas, J. (2020). La importancia de utilizar la Evaluación Formativa y Compartida en la Formación Inicial del Profesorado de Educación Física: Ios Proyectos de 
Aprendizaje Tutorado como ejemplo de buena práctica. Retos. Nuevas tendencias en Educación Fisica, Deporte y Recreación, 37(September), 620-627. https://doi.org/10.47197/retos.v37i37.74193

Massot, I., Dorio, I., \& Sabariego, M. (2014). Estrategias de recogida de información y análisis de la información. En R. Bisquerra (Ed.), Metodología de la investigación educativa (4a ed., pp. 329-366). Madrid: La Muralla.

Navarro, V., \& Jiménez, F. (2012). La mejora en la evaluación formativa de maestros de educación física través de un instrumento de metaevaluación didáctica. Revista Internacional de Ciencias del Deporte, 8(27), 63-79. https://doi.org/10.5232/ricyde2012.02705

Rodríguez, G., Gil, J., \& García, E. (1999). Metodología de la investigación cualitativa (2a). Málaga: Aljibe.

Salinas, D. (2002). ¡Mañana examen! La evaluación: entre la teoría y la realidad. Barcelona: Graó.

Santos, M. (2003). Una flecha en la diana. La evaluación como aprendizaje. Madrid: Narcea.

Santos, M., \& Moreno, T. (2004). ¿El momento de la metaevaluación educativa? Consideraciones sobre epistemología, método, control y finalidad. Revista Mexicana de Investigación Educativa, 9(23), 913-931. Recuperado de http://www.oei.es/evaluacioneducativa/momento_metaevaluacion_educativ a_santos_guerra.pdf

Shön, D. (1998). El Profesional reflexivo: cómo piensan los profesionales cuando actúan. Barcelona: Paidós.

Stake, R. (2006). Multiple case study analysis. New York: Guilford.

Stufflebeam, D. (2001). The metaevaluation imperative. American Journal of Evaluation, 22(2), 183-209. https://doi.org/10.1177/109821400102200204

Taylor, S., \& Bogdan, R. (1987). Introducción a los métodos cualitativos de investigación. Barcelona: Paidós.

Trigueros-Cervantes, C., Rivera-García, E., \& De la Torre-Navarro, E. (2012). La evaluación en el aula universitaria: del examen tradicional a la autoevaluación. Revista Internacional de Medicina y Ciencias de la Actividad Fisica y del Deporte, 12(47), 473-491.

Tristán, J. (2010). La relación de la planificación en la interacción sobre los comportamientos del profesorado principiante y con experiencia así como la de sus alumnos en un estudio de casos. (Tesis Doctoral), Universidad de Murcia, Murcia.

Tsangaridou, N. (2005). Classroom Teachers' Reflections on Teaching Physical Education. Journal of teaching in physical education, 24(1), 24-50. https://doi.org/10.1123/jtpe.24.1.24

Tsangaridou, N., \& O'Sullivan, M. (1997). The Role of Reflection in Shaping Physical Education Teachers' Educational Values and Practices. Journal of Teaching in Physical Education, 17(1), 2-25. https://doi.org/10.1074/jbc.M409740200

Van Manen, M. (1977). Linking ways of knowing with ways of being practical. Curriculum Inquiry, 6(3), 205-228. https://doi.org/10.2307/1179579

Velázquez, R., \& Hernández, J. (2004). Evaluación en educación y evaluación del aprendizaje en educación física. En J. Hernández \& R. Velázquez (Eds.), La evaluación en educación física. Investigación y práctica en el ámbito escolar (pp. 11-47). Barcelona: Graó. 
Woods, P. (1987). La escuela por dentro. Etnografía en la investigación cualitativa. Madrid: Paidós.

Zeichner, K. (1998). Los profesores como profesionales reflexivos y la democratización de la reforma escolar. En Volver a pensar la educación (Vol. II). Prácticas y discursos educativos. (Congreso Internacional de Didáctica) (2a, pp. 385-398). Madrid: Morata.

Zeichner, Kenneth. (2008). Uma análise crítica sobre a "reflexâo" como conceito estruturante na formaçâo docente. Revista educaçâo e sociedade, 29(103), 535-554. https://doi.org/10.1590/S0101-73302008000200012

Número de citas totales I Total references: 42 (100\%)

Número de citas propias de la revista / Journal's own references: 1 (2.3\%)

Rev.int.med.cienc.act.fís.deporte - vol. 21 - número 83 - ISSN: 1577-0354 